\title{
Rational for the Intradermal Therapy (Mesotherapy) in Sport Medicine: From Hypothesis to Clinical Practice
}

ISSN: 2577-1914

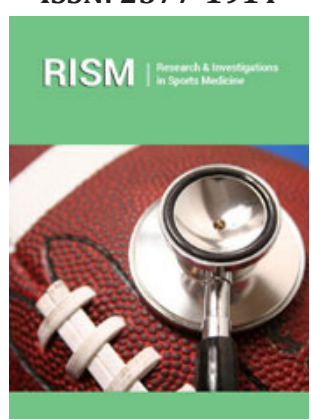

*Corresponding author: Massimo Mammucari, Italian society of Mesotherapy, Italy

Email: massimo.mammucari@libero.it

Submission: 監 October 23, 2019

Published: 眥 November 12, 2019

Volume 5 - Issue 4

How to cite this article: Mammucari $\mathrm{M}^{*}$ Maggiori E, Antonaci L, Fanelli R, Gallo A, et al. Rational for the Intradermal Therapy (Mesotherapy) in Sport Medicine: From Hypothesis to Clinical Practice. Res Inves Sports Med. 5(4). RISM.000619.2019. DOI: 10.31031/RISM.2019.05.000619

Copyright@ Massimo Mammucari, This article is distributed under the terms of the Creative Commons Attribution 4.0 International License, which permits unrestricted use and redistribution provided that the original author and source are credited.

\begin{abstract}
Mammucari Massimo*, Maggiori Enrica, Antonaci Luciano, Fanelli Renato, Gallo Alessandra, Guglielmo Costanza, Russo Domenico, Canzona Flora, Dorato Dario, Di Marzo Raffaele, Natoli Silvia, Rocchi Piergiovanni, Cuguttu Anna, Giardini mAnuela, Gallo Rodolfo and Bomprezzi Alessandro
\end{abstract}

Italian society of Mesotherapy, Italy

\begin{abstract}
Many athletes, after a trauma, need a rapid therapeutic and rehabilitative solution to get back to sports activities. Many symptoms can limit rehabilitation, such as inflammation, oedema, muscle contraction, and pain. These symptoms can be treated with appropriate therapies. Efficacy and tolerability data have been reported with the application of mesotherapy in many local pain syndromes. Is there a rationale for the use of this technique to manage athletes who need rapid recovery after a sports trauma? For many years it has been believed that the pharmacological action alone was able to explain the clinical effects, recently a hypothesis of a complex "dermal interaction" has been advanced which justifies the use of intradermal therapy even after a sports trauma. Remarks from the Italian Society of Mesotherapy for sports medicine specialists are reported.
\end{abstract}

\section{Introduction}

Mesotherapy is a pharmacological technique that, through a series of microinjections, allows the inoculation of active principles in the dermis [1]. Drugs injected with this technique reach the underlying tissues at higher concentrations than those obtained with intramuscular administration. Preclinical studies have shown that drug concentration in the skin, muscles and joints underlying the injection site is higher than after intramuscular administration [24]. Furthermore, it has been demonstrated [5,6] that the production of antibody response is greater if the antigen is inoculated intradermal with respect to the intramuscular route. Some unpublished clinical results $[7,8]$ confirm that a drug remains longer in the underlying tissues after intradermal injection (up to $2 \mathrm{~mm}$ ) than in the deeper injection (greater than $10 \mathrm{~mm}$ ). Similar results have been demonstrated in patients with the administration of human follicle stimulating hormone ( $\mathrm{rhFSH}$ ) injected at a depth of 1-2mm compared to the deeper administration (10-13mm) [9]. These data suggest that the skin constitutes a "deposit" that modulates the spread of drugs in the underlying tissues with the potential advantages of a lower dose requirement and a lower risk of systemic interactions [1]. Furthermore, the microtrauma induced by the needle and distension of the skin tissues due to the injected liquid would stimulate the reactions of the reflex nerves with an activation of the gate control [10]. Also, the chemical-physical properties of the injected liquid can induce some local reactions $[11,12]$. Finally, a glial dermal network able to actively participate in the regulation of local pain, could be involved [13]. All these data suggest that intradermal therapy is the result of a series of "meso-dermal reactions" partly due to the pharmacological effect but enhanced by other intradermal mechanisms.

This hypothesis would be the basis of the short- and medium-term analgesic effects reported in real practice $[14,15]$. Intradermal therapy is a medical technique that can be practiced, both to reduce acute symptoms and to facilitate post-traumatic rehabilitation, alone or in combination with standard therapies. Some experimental data indicate that intradermal therapy in acute localized pain allows results comparable to oral or intramuscular systemic therapy [16-18]. Even when localized acute pain required intervention in an emergency room, intradermal therapy achieved results that were no less than intravenous administration [19]. 
These data suggest an advantage in terms of lower pharmacological systemic interactions for less use of local drugs.

Obviously, the injected drugs must be selected based on the pathophysiology of the symptom (inflammation, oedema, pain, muscle contraction), and based on the patient's trauma (tightness, strain, tear, rupture) (Figure 1). In any case, the sport specialist must obtain the patient's informed consent to apply intradermal therapy and also to share treatment and rehabilitation plan. For this purpose, the Italian society of mesotherapy has validated an information sheet to facilitate communication between doctor and patient to share the benefits and limits of this technique

https://www.societadimesoterapia.it/images/foglioinformativo-sim-2019.pdf

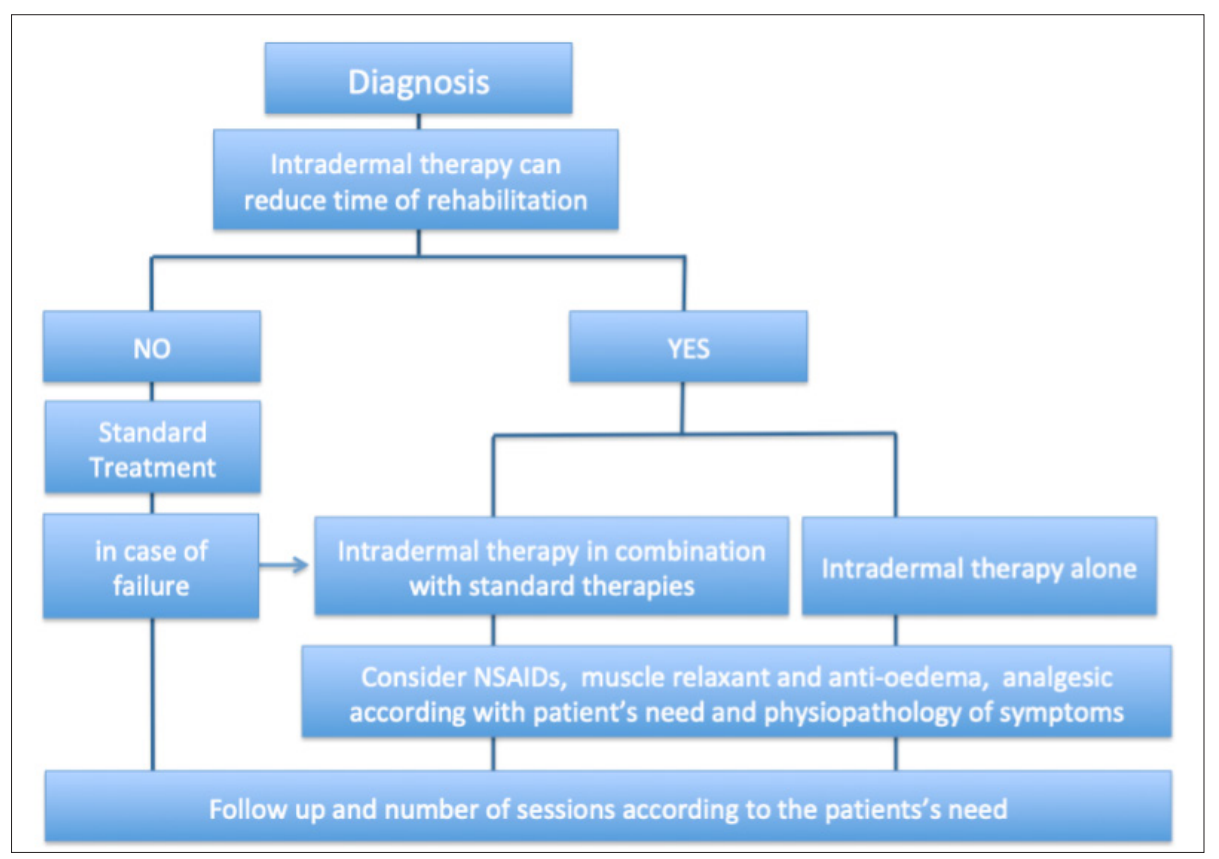

Figure 1: Mesotherapy's Algorithm for sports trauma.

The figure shows the proposed algorithm treatment with intradermal therapy. The informed consent must be collected before applying the therapy

\section{Conclusion}

In many countries of the world, various localized pain syndromes are treated with intradermal therapy, both to reduce healing time and to reduce the risk of systemic drug interactions. Of course, additional randomized clinical trials are needed to compare the utility of mesotherapy with standard therapies or in combination with them in sports trauma. However, the beneficial potential of intradermal therapy could become a standard resource to help athletes recover efficiently after a trauma. The potential benefit of intradermal therapy plays a role both as a model for clinical research and the study of the intradermal effects of many drugs used today (muscle relaxants, anti-inflammatories drugs, vasodilators, analgesics, anaesthetics, phlebotropics) and also to help athletes recover efficiently after a trauma. If the pharmacological mechanism is assisted by reflexological mechanisms, local chemical-physical effects and their interactions with dermal nerve centres, the recommendations of the Italian Mesotherapy society to use a single drug rather than mixtures of drugs to reduce symptoms appear even more convincing. The choice of therapy must be based on the diagnosis, the physiopathological nature of the damage and the characteristics of the individual athlete. A personalized medicine is an indispensable criterion for applying the intradermal-therapy (mesotherapy) in the field of sports medicine.

\section{Disclosure}

The authors declare that they have not conflicted with the content of the article. No fees were received for the preparation of the article. All authors agree with the content.

\section{References}

1. Mammucari M, Gatti A, Maggiori S, Bartoletti CA, Sabato AF, et al. (2011) Mesotherapy, definition, rationale and clinical role: A consensus report from the Italian society of mesotherapy. Eur Rev Medical and Pharmacol Sci 15(6): 682-694.

2. Binaglia L, Marconi P, Pitzurra (1981) Absorption of Na ketoprofen administered intradermally. J of Mesother 1: 85-91.

3. Binaglia L, Marconi P, Pitzurra (1981) The diffusion of intradermally administered procaine. J of Mesother 1: 15-28.

4. Pitzurra M, Cavallo R, Farinelli S, Sposini T, Cipressa T, et al. (1981) On the intradermal inoculation of antibiotics: Some experimental data. J of Mesother 1: 9-14.

5. Pitzurra M, Marconi P (1981) Immunogenesis and mesotherapy: the immunoresponse to antigens inoculated intradermally. J of Mesother 1: 9-14.

6. Coudeville L, Andre P, Bailleux F, Weber F, Plotkin S, et al. (2010) A new approach to estimate vaccine efficacy based on immunogenicity data applied to influenza vaccines administered by the intradermal or intramuscular routes. Hum Vaccin 6(10): 841-848. 
7. Kaplan JA, Coutris G (1992) Mesoscintigraphy and proposal of a unified theory of lamésothérapie. In: Bulletin 5 of the Communications of the $6^{\text {th }}$ International Congress of Mesotherapy, Paris, France, p. 2-4.

8. Mrejen DS (1992) Pharmacokinetics and depth of injections in mesotherapy. In: Bulletin 5 of the Communications of the $6^{\text {th }}$ International Congress of Mesotherapy, French Society of Mesotherapy, Paris, Brussels, Belgium, p. 13-14.

9. Hsu CC, Kuo HC, Hsu CT, Gu Q (2011) Abdominal mesotherapy injection extended the absorption of follicle stimulating hormone. Fertility and Sterility 95(6): 2134-2136.

10. Crenna P, Mancia P (1981) Reflex actions in mesotherapy. J of Mesother 1: 29-40.

11. Koyucu RG, Demirci N, Yumru AE, Salman S, Ayanoğlu YT, et al. (2018) Effects of intradermal sterile water injections in women with low back pain in labor: A randomized, controlled, clinical trial. Balkan Med J 35: 148-154.

12. Lindahl O (1961) Experimental skin pain induced by injection of watersoluble substances in humans. Acta Physiol Scand Suppl 179: 1-89.

13. Abdo H, Calvo-Enrique L, Lopez JM, Song J, Zhang MD, et al. (2019) Specialized cutaneous Schwann cells initiate pain sensation. Science 365(6454): 695-699.
14. Mammucari M, Gatti A, Maggiori S, Sabato AF (2012) Role of mesotherapy in musculoskeletal pain: Opinions from the Italian society of mesotherapy. Evidence-based Complementary and Alternative Medicine 2012: 436959.

15. Paolucci T, Bellomo RG, Centra MA, Giannandrea N, Pezzi L, et al. (2019) Mesotherapy in the treatment of musculoskeletal pain in rehabilitation: The state of the art. J Pain Res 12: 2391-2401.

16. Costantino C, Marangio E, Coruzzi G (2011) Mesotherapy versus systemic therapy in the treatment of acute low back pain: A randomized trial. Evid Based Complement Alternat Med p: 317183.

17. Chen L, Li D, Zhong J, Qiu B, Wu X, et al. (2018) Therapeutic Effectiveness and Safety of Mesotherapy in Patients with Osteoarthritis of the Knee. Evid Based Complement Alternat Med 2018: 6513049 DOI: https://doi. org/10.1155/2018/6513049.

18. Saggini R, Di Stefano A, Dodaj I, Scarcello L, Bellomo RG, et al. (2015) Pes anserine bursitis in symptomatic ostearthritis patients: a mesotherapy treatment study. J Altern Complement Med 21(8): 480-484.

19. Kocak AO (2019) Intradermal mesotherapy versus systemic therapy in the treatment of musculoskeletal pain: A prospective randomized study. Am J Emerg Med S0735-6757(19): 30146-30149. 